\title{
Access to the Left Atrium for Delivery of Radiofrequency Ablation in Young Patients: Retrograde Aortic vs Transseptal Approach
}

\author{
I.H. Law,* P.S. Fischbach, S. LeRoy, T.R. Lloyd, A.P. Rocchini, M. Dick \\ University of Michigan Congenital Heart Center, C. S. Mott Children's Hospital and the Department of Pediatrics, University \\ of Michigan Health Systems, Ann Arbor, MI 48109, USA
}

\begin{abstract}
Reported experience with the transseptal approach to the left atrium for delivery of radiofrequency energy in the young patient is limited. To compare two approaches for radiofrequency ablation (RFA) in the left atrium we reviewed our experience from January 1, 1991, through February 1, 1999, in 154 procedures performed on 136 patients (mean age 12.2 years). The patients were grouped by either the retrograde aortic route $(\mathrm{R}, n=30)$ or the transseptal atrial route $(\mathrm{T}, n=106)$. No significant differences were found in age, weight, height, supraventricular tachycardia cycle length, or electrocardiograph characteristics (manifest vs concealed accessory pathway) between the two approaches. Comparison of the transseptal group to the retrograde aortic group revealed a significant difference in the number of catheters (mean $=4 \mathrm{R}$ vs $3 \mathrm{~T}, p<0.0001$ ), total fluoroscopic time (71.3 $\min \mathrm{R}$ vs $43.0 \min \mathrm{T}, p=0.0007$ ), diagnostic fluoroscopic time (40.2 min $\mathrm{R}$ vs $16.6 \mathrm{~min} \mathrm{~T}$, $p<0.0001$ ), ablation fluoroscopic time (44.7 min $\mathrm{R}$ vs $25.3 \min \mathrm{T}, p=0.019)$, and procedure time (5.0 hours $\mathrm{R}$ vs 4.1 hours $\mathrm{T}, p<0.0001)$. No significant difference was found in success rate, number of radiofrequency applications, or major complication rate. These data suggest that although outcomes and major complication rates are similar for the two groups, the use of fewer catheters and shorter fluoroscopic times warrant consideration of the transseptal atrial approach in young patients.
\end{abstract}

Key words: Radiofrequency ablation - Supraventricular tachycardia — Transseptal technique

During the past decade, radiofrequency (RF) ablation of both abnormal conducting pathways and abnormal ecto-

\footnotetext{
*Current address: The University of Iowa, Iowa City, IA, USA Correspondence to: M. Dick II, F1310 Box 0204, University of Michigan Medical Center, 1500 East Medical Center Drive, Ann Arbor, MI 48109-0204, USA
}

pic foci for the treatment of several types of atrial arrhythmias has emerged as a safe and effective technique in older patients $[2,3,4,8,10,15]$. This technique, using special adaptations such as composite catheters [3], smaller caliber catheters, long vascular sheaths [14], and the transhepatic technique [7], has been broadened to include children of all sizes with both normal and structurally abnormal hearts [16]. The purpose of this report is to evaluate the transseptal approach to left atrial arrhythmias for delivery of radiofrequency energy and to compare this technique to the retrograde approach in young patients.

\section{Methods}

Since January 1, 1991, 136 children and young patients aged 13 months to 28 years have undergone 154 mapping and ablation procedures of either a left-sided accessory pathway $(n=150)$ or a left-sided atrial focus $(n=4)$ at the C. S. Mott Children's Hospital, University of Michigan Health Systems. Prior to October 1992, all left-sided pathways were approached via the retrograde aortic route. Since then, all but 4 left-sided pathways have been approached via the transseptal route. The 4 patients with left-sided atrial foci have been approached through the transseptal route. All patients were studied in the postabsorptive state; deep sedation was achieved with general anesthesia. The transseptal puncture was accomplished with pediatric or adult-length transseptal needles (Daig, Inc., Minnetonka, MN, USA) and standard Mullins transseptal sheaths (CR Bard, Billerica, MA, USA) of 6 Fr to $8 \mathrm{Fr}$ or long sheaths designed specifically for radiofrequency ablation (Daig). Three to five electrophysiologic catheters were placed in the right and left femoral veins as well as the right femoral artery and the left subclavian vein as necessary. Standard atrial and ventricular programmed extrastimulation and endocardial mapping techniques were used to induce each tachyarrhythmia, identify its mechanism, and locate the origin. In those patients who underwent the transseptal approach, the steerable radiofrequency ablation catheter was initially placed in the coronary sinus for mapping of the left atrioventricular groove. Radiofrequency energy was delivered through 5 , 6, or $7 \mathrm{Fr}$ catheters with an oversized $4-\mathrm{mm}$ electrode at the catheter tip. The transseptal approach to the left atrium was used in 106 patients and the retrograde aortic approach into the left ventricle in 30 patients. Prior to October 1991, 3000 units of heparin was administered intravenously every 45 to 60 minutes for anticoagulation. After October 1991, the 
Table 1. Patient characteristics of the two approaches

\begin{tabular}{|c|c|c|c|c|c|c|}
\hline \multirow[t]{2}{*}{ Characteristic } & \multicolumn{2}{|c|}{ Entire group analysis } & \multirow[t]{2}{*}{$p$ value } & \multicolumn{2}{|l|}{ Subset analysis } & \multirow[t]{2}{*}{$p$ value } \\
\hline & $\begin{array}{l}\text { Retrograde } \\
\text { aortic approach } \\
(n=30)\end{array}$ & $\begin{array}{l}\text { Transseptal } \\
\text { approach } \\
(n=106)\end{array}$ & & $\begin{array}{l}\text { Retrograde } \\
\text { aortic approach } \\
(n=30)\end{array}$ & $\begin{array}{l}\text { Transseptal } \\
\text { approach } \\
(n=30)\end{array}$ & \\
\hline Gender-female & 8 & 44 & NS & 8 & 15 & NS \\
\hline Age (years) & $13.1 \pm 4.4$ & $11.6 \pm 5.3$ & NS & $13.1 \pm 4.4$ & $12.8 \pm 4.6$ & NS \\
\hline Height $(\mathrm{cm})$ & $155.4 \pm 22.4$ & $145.6 \pm 26.4$ & NS & $155.4 \pm 22.4$ & $151.5 \pm 21.2$ & NS \\
\hline Weight $(\mathrm{kg})$ & $53.0 \pm 21.5$ & $45.4 \pm 24.0$ & NS & $53.0 \pm 21.5$ & $49.6 \pm 21.7$ & NS \\
\hline SVT cycle length (seconds) & $325.3 \pm 43.9$ & $310.1 \pm 65.0$ & NS & $325.3 \pm 44.0$ & $307.5 \pm 65.0$ & NS \\
\hline Concealed $(\%)$ & $9(30)$ & $49(46)$ & NS & $9(30)$ & $13(43)$ & NS \\
\hline Manifest (\%) & $21(70)$ & $54(52)$ & NS & $21(70)$ & $16(53)$ & NS \\
\hline
\end{tabular}

NS, not statistically significant; SVT, supraventricular tachycardia.

activated coagulation time (ACT) was measured every 30 to 45 minutes and heparin was given to maintain the ACT equal to or greater than 300 seconds. For the patients undergoing the transseptal technique, administration of heparin was delayed until the left artery had been entered.

To compare the efficacy and safety of these two approaches, we recorded the number of catheters required, the number of radiofrequency applications required, total fluoroscopic time and the fluoroscopic time devoted to the ablation procedure (as opposed to diagnostic and mapping procedures), the success rate, and complications. A successful outcome was defined as no inducible supraventricular tachycardia (SVT) at the conclusion of the procedure. Major complications were defined as complications of the radiofrequency ablation procedure that required intervention or produced a persistent (but not necessarily permanent) deficit. Minor complications were defined as self-limiting or nonprogressive. To account for improvement in technique with experience, a subset analysis was performed comparing the first 30 patients who underwent either approach. A second subset of the last 30 transseptal patients was compared to the first 30 transseptal patients to test if differences, if any, related to the two different techniques were maintained over time. The categorical variables were compared by Chi-square analysis and the continuous variables by Student's $t$-test. A $p$ value $\leq 0.05$ was taken to denote statistical significance.

\section{Results}

Patient characteristics and pathway and tachycardia features are summarized in Table 1. All but 19 of the 136 patients were 18 years or younger. Ninety-four percent $(128 / 136)$ of the patients had orthodromic atrioventricular reentrant tachycardia, 3\% (4/136) had automatic atrial tachycardia, $1 \%(2 / 136)$ had antidromic atrioventricular reentrant tachycardia, and 1 patient each had either persistent junctional reciprocating tachycardia or both antidromic and orthodromic reentrant tachycardia. Outcome comparison by procedure is summarized in Table 2 and by patient in Table 3 . The overall success rate for both approaches was 96\% (131/136) of patients and 93\% $(143 / 154)$ of procedures. In the subset analysis of the first 30 patients in each approach the success rate was 97\% (58/60) of patients and 92\% (66/72) of procedures.
The overall complication rate (major complications and minor complications) was not significantly different between the two approaches. The combined major complication rate for both approaches was $5.1 \%(11 / 136)$ of patients and $4.5 \%$ (7/154) of procedures. The combined major complication rate for the first 30 patients in each approach was 5\% (3/60) of patients and 4.2\% (3/72) of procedures.

When we compared the first 30 patients to undergo each approach, as well as the entire population by each approach, there was no difference in sex distribution, mean age, and mean weight, mean height, and mean SVT cycle length between the retrograde group and the transseptal group. There was also no significant difference between the two approaches in the number of patients with either manifest or concealed accessory pathways (Table 2).

The median number of catheters necessary for successful ablation in the transseptal group $(n=3)$ was significantly less $(p \leq 0.001)$ than the median number used in the retrograde approach $(n=4)$. The number of RF energy applications was similar for both approaches in the entire group analysis and in the subset analysis. When comparing the length of the procedures between the two approaches for all 136 patients, the retrograde aortic approach required significant longer procedure times $(p<0.0001)$. Procedure time was also longer in the retrograde group but not significantly so between the two subsets.

\section{Fluoroscopic Time}

Total fluoroscopic time (diagnostic and ablation fluoroscopic times combined), a measure of radiation exposure, was significantly less in the group approached transseptally $(p=0.007)$ and trended toward significance in the subset analysis. This difference also held 
Table 2. Outcome comparison by procedure between the two approaches

\begin{tabular}{|c|c|c|c|c|c|c|}
\hline & \multicolumn{2}{|c|}{ Entire group analysis } & \multirow[t]{2}{*}{$p$ value } & \multicolumn{2}{|l|}{ Subset analysis } & \multirow[t]{2}{*}{$p$ value } \\
\hline & $\begin{array}{l}\text { Retrograde aortic } \\
\text { approach } \\
(n=36)\end{array}$ & $\begin{array}{l}\text { Transseptal } \\
\text { approach } \\
(n=118)\end{array}$ & & $\begin{array}{l}\text { Retrograde aortic } \\
\text { approach } \\
(n=36)\end{array}$ & $\begin{array}{l}\text { Transseptal } \\
\text { approach } \\
(n=36)\end{array}$ & \\
\hline Number of successes (\%) & $31(86)$ & $112(95)$ & NS & $31(86)$ & $35(97)$ & NS \\
\hline Number of catheters & $4.1 \pm 0.5$ & $3.1 \pm 0.8$ & $<0.0001$ & $4.1 \pm 0.5$ & $2.8 \pm 1.1$ & $<0.0001$ \\
\hline Procedure time (hours) & $5.02 \pm 2.03$ & $4.07 \pm 0.47$ & $<0.0001$ & $5.02 \pm 2.03$ & $4.8 \pm 2.8$ & NS \\
\hline Total fluoroscopic time (minutes) & $71.3 \pm 61.7$ & $43.0 \pm 35.1$ & 0.007 & $71.3 \pm 61.7$ & $46.8 \pm 41.3$ & NS \\
\hline $\begin{array}{l}\text { Diagnostic fluoroscopic } \\
\text { time (minutes) }\end{array}$ & $40.2 \pm 47.6$ & $16.6 \pm 8.1$ & $<0.0001$ & $40.2 \pm 47.6$ & $14.8 \pm 3.1$ & 0.0024 \\
\hline $\begin{array}{l}\text { Ablation fluoroscopic } \\
\text { time (minutes) }\end{array}$ & $44.8 \pm 61.1$ & $25.3 \pm 33.1$ & 0.03 & $44.8 \pm 61.1$ & $32.0 \pm 40.3$ & NS \\
\hline No. of RF applications & $11 \pm 12$ & $9 \pm 10$ & NS & $11 \pm 12$ & $12 \pm 13$ & NS \\
\hline No. of major complications (\%) & $1(2.8)$ & $6(5.1)$ & NS & $1(2.8)$ & $2(5.6)$ & NS \\
\hline No. of minor complications $(\%)$ & $6(17)$ & $6(5.1)$ & 0.02 & $6(17)$ & $1(2.8)$ & 0.047 \\
\hline
\end{tabular}

NS, not statistically different; RF, radiofrequency.

Table 3. Outcome comparison by patient between the two approaches

\begin{tabular}{|c|c|c|c|c|c|c|}
\hline & \multicolumn{2}{|c|}{ Entire group analysis } & \multirow[t]{2}{*}{$p$ value } & \multicolumn{2}{|l|}{ Subset analysis } & \multirow[t]{2}{*}{$p$ value } \\
\hline & $\begin{array}{l}\text { Retrograde aortic } \\
\text { approach } \\
(n=30)\end{array}$ & $\begin{array}{l}\text { Transseptal } \\
\text { approach } \\
(n=106)\end{array}$ & & $\begin{array}{l}\text { Retrograde aortic } \\
\text { approach } \\
(n=30)\end{array}$ & $\begin{array}{l}\text { Transseptal } \\
\text { approach } \\
(n=30)\end{array}$ & \\
\hline Number of successes (\%) & $29(97)$ & $102(96)$ & NS & $28(93)$ & $29(97)$ & NS \\
\hline No. of major complications (\%) & $1(3.4)$ & $6(5.7)$ & NS & $1(3.3)$ & $2(6.7)$ & NS \\
\hline No. of minor complications (\%) & $6(20)$ & $5(4.7)$ & 0.014 & $6(20)$ & $1(3.3)$ & 0.044 \\
\hline
\end{tabular}

NS, not statistically different.

when comparing the ablation fluoroscopic time (fluoroscopic time required during the ablation portion of the study) $(p=0.03)$. Diagnostic fluoroscopic times were significantly less for the transseptal approach in both the entire group analysis $(p<0.0001)$ and the subset analysis $(p=0.0024)$.

\section{Major Complications}

Although the number of major complications in the transseptal group was greater than that in the retrograde aortic group, there was no significant difference between the two complication rates when compared by procedure or by patient in either analysis. The major complication in the retrograde aortic group was femoral artery occlusion in an 18-year-old man; this patient had multiple pathways (left and right) as well as atrioventricular nodal reentry tachycardia. Because of symptomatic claudication noted several weeks after this procedure, he received a common iliac-femoral artery bypass.
In the transseptal group major complications occurred in six patients; all but one was self-limited or treated without sequelae. Perforation of the posterior wall of the left atrium occurred in three patients (ages 1.4 years, 8.6 years, and 10.7 years), requiring pericardiocentesis in only one. The 10.7-year-old girl also had a transient ischemic attack 1 week later that cleared spontaneously within 2 hours. An 11.6-year-old girl required a chest tube for a left pneumothorax resulting from an additional catheter placed in the left subclavian vein for coronary sinus mapping. A 6.5 -year-old boy had a traumatic mitral valve injury during his second electrophysiology study and RF ablation procedure for a left free wall accessory pathway. Mild mitral regurgitation was noted by echocardiogram after the second procedure that progressed to moderate to severe regurgitation over 2 months requiring surgical valvuloplasty. A 14-year-old patient with automatic atrial tachycardia had a midbrain arterial occlusion with associated findings by examination (diplopia) and magnetic resonance imaging. The findings completely resolved over the following 2 
months. Only one patient in each group required surgical intervention.

\section{Minor Complications}

In contrast to the major complication rate, the minor complication rate was significantly less in the transseptal approach when analyzed by both patients and procedures in the total group ( $p=0.014$ and $p=0.02$, respectively) and in the subset analysis $(p=0.04$ and $p=0.05$, respectively). Minor complications in the retrograde aortic approach included atelectasis (age 4.7 years) and diminished pulse (age 18.8 years), pressure sore in two patients (ages 14.2 and 17.9 years), and trivial to mild aortic insufficiency by auscultation in three patients (ages 9.5, 16.7, and 17.9 years; two of these patients have been previously reported [13]). Minor complications of the transseptal approach included transient complete heart block (age 14.7 years), air embolus to the right coronary artery with transient ST segment changes in two patients (ages 5.9 and 12.9 years), and femoral hematoma (age 3.9 years). Two other minor complications that occurred during the transseptal approach not related to the transseptal technique were transient hair loss (age 15.3 years) and transient bronchospasm during adenosine administration (age 20.3 years).

There were no significant differences in all these measures when the data from the first 30 transseptal patients were compared to the data from the last 30 transseptal patients.

\section{Discussion}

This study confirms the equivalency of the transseptal approach to the retrograde aortic approach for RF ablation in a pediatric population. The transseptal approach to the left heart has been extensively and safely employed in pediatric patients with congenital heart disease for two decades not only for passage through the native atrial septum but also through surgically placed baffles and patches $[1,5,6]$. In our experience, the transseptal approach for RF energy application in the left heart requires fewer catheters and less fluoroscopic time, with no significant difference in the major complication rate in comparison to the retrograde aortic approach.

Several investigators have reported their experience with both the retrograde aortic and transseptal approach for RF ablation. Minich and colleagues [13] reported an approximate 30\% incidence of Doppler detected aortic regurgitation following the retrograde aortic approach to left-sided pathways in 44 young patients undergoing RF ablation for supraventricular tachycardia, possibly related to the duration of time the ablation catheter crossed the aortic valve. Lau and colleagues [11] investigated the incidence of both atrioventricular and semilunar valvar regurgitation following RF ablation on 65 consecutive patients, 33 with left-sided pathways. All left-sided pathways were approached transseptally; 1 patient (1-month old with two accessory pathways) developing new mitral regurgitation. None of the patients developed new aortic regurgitation. Our report suggests equal efficacy and complication rate between the two approaches. However, as noted in our current study and in Lau et al.'s study, care is needed when crossing the atrial septum to avoid injury to the mitral valve in small children.

Vora and colleagues [17] performed a similar retrospective study on a smaller number of pediatric patients $(n=49)$ with left-sided accessory pathways. In contrast to our study, they found significantly shorter fluoroscopic times in the retrograde aortic route group when compared to the transseptal approach (38 vs $61 \mathrm{~min}$ ). These shorter fluoroscopic times in the retrograde aortic approach may have been associated with their previous experience with this approach in their adult population. Because we routinely perform left heart catheterization on patients with congenital heart disease through the transseptal approach, our experience allows incorporation of the transseptal technique into RF ablation procedures. Furthermore, in contrast to our experience, Vora and associates [17] found no difference in the number of catheters or complications. Vora et al.'s study demonstrates that both approaches in the pediatric population are safe but it overlooks the advantage of reducing the number of catheter sheaths by deploying the steerable ablation catheter into the coronary sinus for mapping prior to transseptal entry into the left atrium for RF ablation. Transferring the venous mapping catheter to an arterial sheath for ablation could also take advantage of this dual use of the ablation catheter both for mapping in the coronary sinus and for ablation by the retrograde approach. However, an arterial sheath would be needed in the retrograde approach, jeopardizing the femoral artery. Finally, we recognize that the number of catheters may be predicated on the operator choice rather than by the approach. Nonetheless, the fewer the number of catheters inserted into the child's circulation, the less exposure to potential adverse events.

Lesh and associates [12] compared the transseptal atrial and retrograde aortic approaches in 106 patients whose mean age was 33 years. With an overall ablation success rate of $96.2 \%$, no difference was found between the two approaches with respect to success, complication rate $(6.7 \%$ retrograde vs $6.1 \%$ transseptal), or fluoroscopic times for the two procedures. This study emphasizes the favorable success rate of both approaches in older, larger sized patients and emphasizes the similar complication rates between Lesh et al.'s adult population and our pediatric population. Similar recent success rates (91-96\%) for left-sided pathways in children have been 
noted by the Pediatric Radiofrequency Ablation Registry (J. Kugler personal communication).

Several factors may contribute to the different complication rate between the two approaches in our study. First, the retrograde approach was our initial approach and thus served as an early learning curve for the RF ablation procedure. Second, the larger sized patients and the higher incidence of manifest pathways in this initial retrograde group reflect our early practice of reserving ablation for larger and older patients who most convincingly had an atrioventricular accessory pathway as the mechanism of their tachycardia. The subset analysis comparing the first 30 patients and 36 procedures for each approach was performed to offset this learning curve effect. The patient characteristics were even more similar between these two smaller subsets and did not change over time. However, significant differences favoring the transseptal approach were noted in the number of catheters, ablation fluoroscopic time, and minor complications.

Third, the observation of Doppler detected aortic regurgitation and the development of mild aortic insufficiency by auscultation in three patients following the retrograde procedure, as well as the femoral artery injury in one 18-year-old man, led us to explore the transseptal approach. Nonetheless, it should be noted that none of the patients with mild aortic regurgitation by auscultation or by Doppler have experienced progression over a 6- to 8 -year follow-up. Furthermore, congruent with the experience of others [11], no patients in the transseptal group developed aortic regurgitation detected either by auscultation or by Doppler.

Fourth, six patients who underwent the transseptal route experienced major complications. Five were transient: left atrial perforation in two, neuroembolic events in two, and pneumothorax in one. Although these events are not negligible, they are routinely sought after the transseptal puncture (i.e., by echocardiogram and chest $\mathrm{x}$-ray), are usually self-limited or readily managed when identified promptly, and are known potential complications of invasion of the systemic circulation during either cardiac catheterization or heart surgery. The most serious event occurred in the 6.5-year-old boy who developed progressive mitral regurgitation. On review, it was noted that the transseptal trochar was advanced inferiorly and posteriorly across the atrial septum, directing the transseptal sheath and ablation catheter tangential to the mitral annulus, toward the posterior mitral valve leaflet, most likely accounting for the injury to the mitral valve.

Overall, the majority of the complications were not related to the RF current per se. On the other hand, the two neuroemboli were likely linked to the generation of RF current.

Radiation exposure is a particular concern in young patients. Since 1990 and 1991, before the use of the transseptal route, there has been $20 \%$ decrease in total fluoroscopy time (mean of 50 to mean of 40 minutes) among patients undergoing RF ablation of left-sided pathways. Knowledge transfer of the ablation technique developed during the retrograde aortic era, as well as computer-assisted recording systems and improved catheters and sheaths, coincided with adoption of the transseptal route. Furthermore, the reduction in mean fluoroscopy time (40 minutes in 1992 and 1993 to 30 minutes in 1995 and 1996) accompanied the introduction of the transseptal route. As recently reported, total radiation exposure to patients undergoing RF ablation is no greater than the radiation dose (rems) received by young patients undergoing diagnostic and interventional cardiac catheterization. This equilvalency is largely achieved by avoiding cineangiography during RF ablation procedures [9].

This study has several limitations. First, the data were collected historically and not contemporaneously. We compared recent experience by the transseptal route to immediately prior historical experience by the retrograde aortic route. Thus, the transseptal method benefited by transfer of knowledge acquired during the retrograde aortic approach era as well as experience gained during heart catheterizations on patients with complex congenital heart disease. The subset analysis comparing the first 30 patients by each approach was performed to minimize the learning curve effect and patient selection bias that occurred in the early procedure years. The transseptal approach, at a minimum, is equal to the retrograde aortic route and appears to result in less radiation exposure and lower total (major and minor) complication rate than the retrograde approach for ablation of left-sided structures in young patients.

\section{Clinical Implications}

We conclude that access to left atrial accessory pathways or ectopic foci can be achieved in young patients with similar outcome through either the transseptal route or retrograde aortic approach. Equivalent success, similar complication rates, and improved fluoroscopy times can be achieved without jeopardizing arterial integrity or aortic valve function; however, attention to the mitral ring and valve is critical when using the transseptal approach.

Acknowledgment. Supported in part by American Heart Association Michigan Affiliate Fellowship Grant No. 98045007 (IHL).

\section{References}

1. Ali Khan MA, Mullins CE, Bash SE, et al. (1989) Transseptal left heart catheterization in infants, children, and young adults. Cathet Cardiovasc Diagn 17:198-201

2. Calkins H, Sousa J, el-Atassi R, et al. (1991) Diagnosis and cure of the Wolff-Parkinson-White syndrome or paroxysmal supraven- 
tricular tachycardias during a single electrophysiologic test [see comments]. N Engl J Med 324:1612-1618

3. Dick M, Law IH, Dorostkar PC, Armstrong B, Reppert C (1996) Use of the His/RVA electrode catheter in children. $J$ Electrocardiol 29:227-233

4. Dick M, O'Connor BK, Serwer GA, LeRoy S, Armstrong B (1991) Use of radiofrequency current to ablate accessory connections in children. Circulation 84:2318-2324

5. Duff DF, Mullins CE (1998) Transseptal left heart catheterization in infants and children. Cathet Cardiovasc Diagn 4:213-223

6. El-Said HG, IFF, Nihill MR, et al. (1998) 18 year experience with transseptal procedures through baffles, conduits, and other intraatrial patches. J Am Coll Cardiol 31(Suppl A):58A

7. Fischbach P, Campbell RM, Hulse E, et al. (1997) Transhepatic access to the atrioventricular ring for delivery of radiofrequency energy. J Cardiovasc Electrophysiol 8:512-516

8. Jackman WM, Wang XZ, Friday KJ, et al. (1991) Catheter ablation of accessory atrioventricular pathways (Wolff-Parkinson-White syndrome) by radiofrequency current [see comments]. $N$ Engl $J$ Med 324:1605-1611

9. Kowalski CA, Dorostkar PC, Miklos JA, et al. (1994) Comparison of radiation dose received during either electrophysiologic study and radiofrequency ablation or cardiac catheterization in children. Circulation 90:I-99

10. Langberg JJ, Chin MC, Rosenqvist M, et al. (1989) Catheter ablation of the atrioventricular junction with radiofrequency energy. Circulation 80:1527-1535
11. Lau YR, Case CL, Gillette PC, et al. (1994) Frequency of atrioventricular valve dysfunction after radiofrequency catheter ablation via the atrial approach in children. Am J Cardiol 74:617618

12. Lesh MD, Van Hare GF, Scheinman MM, Ports TA, Epstein LA (1993) Comparison of the retrograde and transseptal methods for ablation of left free wall accessory pathways. J Am Coll Cardiol 22:542-549

13. Minich LL, Snider AR, Dick MD (1992) Doppler detection of valvular regurgitation after radiofrequency ablation of accessory connections. Am J Cardiol 70:116-117

14. Saul JP, Hulse JE, De W, et al. (1993) Catheter ablation of accessory atrioventricular pathways in young patients: use of long vascular sheaths, the transseptal approach and a retrograde left posterior parallel approach. J Am Coll Cardiol 21:571-583

15. Van Hare GF, Lesh MD, Scheinman M, Langberg JJ (1991) Percutaneous radiofrequency catheter ablation for supraventricular arrhythmias in children. J Am Coll Cardiol 17:1613-1620

16. Van Hare GF, Lesh MD, Stanger P (1993) Radiofrequency catheter ablation of supraventricular arrhythmias in patients with congenital heart disease: results and technical considerations. J Am Coll Cardiol 22:883-890

17. Vora AM, McMahon S, Jazayeri MR, Dhala A (1999) Ablation of atrial insertion sites of left-sided accessory pathways in children: efficacy and safety of transseptal versus transaortic approach. Pediatr Cardiol 18:332-338 